\title{
Lisdexamfetamine in the treatment of adolescents and children with attention-deficit/hyperactivity disorder
}

This article was published in the following Dove Press journal:

Adolescent Health, Medicine and Therapeutics

7 May 2012

Number of times this article has been viewed

Jadwiga Najib ${ }^{1-3}$

'Division of Pharmacy Practice, Arnold and Marie Schwartz College of Pharmacy and Health Sciences, Long Island University, Brooklyn, ${ }^{2}$ Department of Pharmacy, ${ }^{3}$ Department of Psychiatry, St Luke's-Roosevelt Hospital Center, New York, NY, USA
Correspondence: Jadwiga Najib Long Island University, Arnold and Marie Schwartz College of Pharmacy and Health Sciences, Division of Pharmacy Practice, HS BIdg 5th Floor, 75 DeKalb Ave, Brooklyn, NY I I20I, USA

$\mathrm{Tel}+\mathrm{I} 2125238226$

Fax + I 2125238660

Email jadwiga.najib@liu.edu

\begin{abstract}
Attention-deficit/hyperactivity disorder is one of the most common neurobehavioral disorders defined by developmentally inappropriate levels of inattention, hyperactivity, and impulsivity. Symptoms begin in childhood and may persist into adolescence and adulthood. Currently available pharmacological treatment options for attention-deficit/hyperactivity disorder in children and adolescents include stimulants that are efficacious and well tolerated; however, many of these preparations require multiple daily dosing and have the potential for abuse. Lisdexamfetamine dimesylate, the first prodrug stimulant, was developed to provide a longer duration of effect. It demonstrates a predictable delivery of the active drug, d-amphetamine, with low interpatient variability, and has a reduced potential for abuse. A literature search of the MEDLINE database and clinical trials register from 1995-2011, as well as relevant abstracts presented at annual professional meetings, on lisdexamfetamine dimesylate in children and adolescents were included for review. This article presents the pharmacokinetic profile, efficacy, and safety of lisdexamfetamine dimesylate for the treatment of attention-deficit/hyperactivity disorder in children and, more recently, in adolescents.
\end{abstract}

Keywords: lisdexamfetamine dimesylate, prodrug stimulant, attention-deficit and hyperactivity disorders, safety, efficacy, children, adolescents

\section{Introduction}

Attention-deficit/hyperactivity disorder (ADHD) is one of the most common neurobehavioral disorders that pediatricians and child psychiatrists see in practice, and which can profoundly affect the well-being, academic achievement, and social interactions of children. ${ }^{1}$ In late 2011, the American Academy of Pediatrics updated their ADHD diagnosis and evaluation guidelines and expanded the age group, previously 6-12 year olds, to include younger preschool children (ages 4 years and 5 years) and adolescents ( $<18$ years of age). ${ }^{2}$ In the United States, approximately $9 \%$ of children in a representative sample of 8-15 year olds met the criteria for ADHD; ${ }^{3}$ likewise, the occurrence rate in adolescents between 13-18 years of age was approximately $8 \%$ according to the National Comorbidity Survey Adolescent Supplement Replication epidemiologic survey. ${ }^{4}$

The underlying pathophysiology of ADHD has not been clearly identified, although neurobiological, genetic, and environmental factors have been implicated. Since the catecholaminergic neurotransmitter system is associated with executive and cognitive functions, disturbances in the regulation of norepinephrine and dopamine have been implicated in ADHD pathogenesis. ${ }^{5}$ Treatment guidelines published by the American Academy of Child and Adolescent Psychiatry and the American Academy 
of Pediatrics, as well as by international consensus, note the strong evidence for safety and efficacy of stimulant medications in the treatment of childhood and adolescent ADHD. ${ }^{2,6-8}$ Other treatment options for ADHD include alpha-2 adrenergic agonists (eg, clonidine, guanfacine), heterocyclic antidepressants (eg, tricyclic antidepressants, bupropion, venlafaxine), arousal agents/hypothalamic center activators (eg, modafinil), and atomoxetine - a nonstimulant selective norepinephrine reuptake inhibitor.

\section{ADHD management issues in adolescents and children}

ADHD first appears in childhood and manifests as a persistent pattern of inattention, hyperactivity, and impulsivity that is more frequent and severe than typically observed in children at a comparable level of age or development. ${ }^{9}$ The disorder may affect the child's life, leading to low self-esteem; higher injury rates due to accidents; poor performance at school, sports, or after-school activities; and conflict with family, friends, and teachers. ${ }^{1,10}$ Untreated ADHD is associated with risk for school failure or dropout, alcohol and/or substance abuse, teen pregnancies, delinquency, and other behavior and discipline problems. ${ }^{10,11}$ Symptoms of ADHD often persist into adolescence and adulthood and result in pervasive impairments across multiple life domains, including home, school, peers, and extracurricular activities. ${ }^{1,12}$

Stimulants, which include amphetamine derivatives (dextroamphetamine sulfate, mixed amphetamine salts), and nonamphetamines, (methylphenidate and dexmethylphenidate) are available in a variety of immediate and extended release formulations. The immediate release preparations, due to their shorter duration, require more frequent administration, usually two to three times a day. ${ }^{9,13}$ This may result in a need for medication supply and reliance on the school nurse for administration, and has the potential for nonadherence and social stigmatism. ${ }^{14}$ The long-acting oral stimulant preparations are usually dosed once a day and their duration of activity is generally $7-12$ hours, depending on the product. ${ }^{15,16}$ They offer improved adherence and greater dosing convenience; however, concerns about the abuse potential of stimulants exist and have led to the development of newer formulations addressing this issue.

Lisdexamfetamine dimesylate (LDX), the first prodrug stimulant, was developed to provide a longer duration of effect and reduce the potential for abuse. Although ADHD is apparent across the lifespan, the focus of this paper is limited to reviewing LDX - a prodrug of d-amphetamine that has been approved since 2007 - for the treatment of ADHD in children and, more recently, in adolescents (since 2010). Published reports of clinical trials with LDX in children and adolescents with ADHD (ie, $<18$ years of age) were identified in a systematic literature search of MEDLINE (PubMed and EMBASE) from 1995-2011. A search for registered clinical trials using LDX for ADHD within clinicaltrials.gov was also conducted and reviewed. Abstracts presented at the annual meetings of the American Psychiatric Association, the American Academy of Child and Adolescent Psychiatry, and the Canadian Academy of Child and Adolescent Psychiatry were reviewed and included if judged to be relevant.

\section{Pharmacology and pharmacokinetics of LDX}

LDX distinguishes itself from other central nervous system stimulants and long-acting preparations due to its prodrug properties and pharmacokinetic profile. ${ }^{17}$ LDX contains d-amphetamine covalently bonded to L-lysine, and undergoes enzymatic hydrolysis to convert the pharmacologically inactive molecule to the active drug moiety, d-amphetamine, primarily in the blood by red blood cells. ${ }^{18}$ It appears that the high capacity absorption and enzymatic conversion may be responsible for the consistent and reproducible pharmacokinetic profile of LDX. Since the enzymatic process appears to be high-capacity, saturation is unlikely to happen at therapeutic doses; however, at dosages greater than $130-150 \mathrm{mg}$, the levels are attenuated due to saturation of the enzymatic hydrolysis, suggesting reduced potential for toxicity in an overdose. ${ }^{19}$

Following oral administration of LDX, the pharmacokinetic profile of d-amphetamine was reportedly similar in pediatric (aged 6-12 years) and adolescent (aged 13-17 years) ADHD patients compared with healthy adults. ${ }^{20}$ In a study of 18 pediatric patients (aged 6-12 years) following ingestion of LDX as a single oral 30,50 , or $70 \mathrm{mg}$ dose, the time to maximum concentration of the prodrug molecule LDX was 1 hour, while the time to maximum concentration of d-amphetamine was 3.5 hours. Peak d-amphetamine levels were dose-proportional and exhibited low interpatient variability. After oral administration of LDX, the serum elimination halflife of d-amphetamine ranged from 8.6-10.4 hours, consistent with data reported from earlier studies. ${ }^{21}$

Since food does not affect absorption of LDX, the drug may be administered with breakfast or the contents of the capsule may be dissolved in water prior to oral administration. 
In contrast, food has been shown to prolong the time to maximum concentration of d-amphetamine from extended release mixed amphetamine salts (MAS XR) by 2.5 hours compared with the fasted state. Once-daily administration of $\operatorname{LDX}(30,50$, or $70 \mathrm{mg} /$ day) compared with MAS XR at equivalent total d-amphetamine base content $(10,20$, or $30 \mathrm{mg} /$ day, respectively) in children (aged 6-12 years) noted that the time to maximum concentration of LDX was 3.5 times less variable than with the MAS XR. LDX showed low patient-to-patient pharmacokinetic variability, and the release of d-amphetamine was more predictable in patients who took $70 \mathrm{mg}$ LDX than in patients who took $30 \mathrm{mg}$ MAS XR (the equivalent total d-amphetamine base content), suggesting consistent drug delivery among patients. When administered orally, the onset of clinical effect was noted within 2 hours, comparable to the clinical effect observed with MAS XR. ${ }^{22}$ LDX maintained efficacy throughout the 12-hour testing period and more recently was shown to be effective 1.5-13 hours postingestion. ${ }^{23}$

Following biotransformation, LDX is hepatically metabolized and nearly the entire dose is renally eliminated as either amphetamine related compounds or inactive metabolites. LDX has a low potential for drug-drug interactions as it is not metabolized by cytochrome P450 enzymes and thus does not inhibit the majority of these enzymes. However, any interactions with LDX would likely be caused by $\mathrm{d}$-amphetamine and its metabolites. ${ }^{24}$

The biotransformation and not the dissolution of intact LDX appears to be responsible for the rate of delivery of the active metabolite. ${ }^{18}$ Since LDX is a prodrug subject to enzymatic hydrolysis following ingestion, and not a controlled release delivery vehicle, it is not likely to be affected by changes in normal gastrointestinal transit times or variations in gastric acidity. ${ }^{20}$ Variations in gastric $\mathrm{pH}$ have not been shown to affect the absorption of LDX, suggesting that no drug interactions should occur with LDX and medications that lower the gastrointestinal pH. However, interpatient variability in gastric $\mathrm{pH}$ and gastrointestinal motility may affect the metabolism of some long-acting preparations of methylphenidate, and impact the delivery of the delayed release active moiety. ${ }^{25}$ Likewise, acidifying and alkalinizing agents may reduce or increase blood levels of amphetamine from MAS XR preparations, respectively. ${ }^{26}$ The low interpatient variability observed with LDX may not alleviate the need to titrate doses, but may aid in the process of developing a dosing regimen for patients, and reduce the likelihood of achieving either subtherapeutic or supratherapeutic levels. ${ }^{25}$

\section{Efficacy data}

\section{Pediatric}

Efficacy of LDX has been established in several clinical trials in children (aged 6-12 years) who met the Diagnostic and Statistical Manual of Mental Disorders (fourth edition, text revision) criteria $^{27}$ for a diagnosis of the combined or predominantly hyperactive-impulsive subtype of ADHD (Table 1).

The first study by Biederman et al, a Phase II trial, compared LDX with MAS XR and placebo in a laboratory classroom environment and used three standard efficacy scales during observations made over a 12-hour period. ${ }^{22}$ These included the Swanson, Kotkin, Agler, M-Flynn, and Pelham (SKAMP)-Deportment scale - which uses an independent observer to assess classroom symptoms of ADHD, the Permanent Product Measure of Performance - a validated test consisting of age-appropriate math questions, and the Clinical Global Impression (CGI) scale - a validated tool for assessing global improvements in symptoms over time. This three-treatment, three-period, crossover study noted that both LDX 30, 50, or $70 \mathrm{mg}$ and MAS XR 10, 20, or $30 \mathrm{mg}$ significantly improved measures of efficacy compared to placebo on all three scales. Improvements over placebo were observed within 2 hours of the LDX dose, with the greatest effect occurring at approximately 6 hours, and treatment effects were seen at 12 hours postdose, the last assessment time point. A clinically meaningful difference between the two active groups was not demonstrated. A post hoc analysis suggested that treatment with LDX, administered at doses containing equivalent amounts of $d$-amphetamine as MAS $\mathrm{XR}$, resulted in greater improvement in ADHD symptoms as evidenced by the CGI-Improvement assessment. ${ }^{28}$

Biederman et al also evaluated the efficacy of LDX 30, 50, and $70 \mathrm{mg}$ in a double-blind, randomized, placebo-controlled, Phase III study involving 290 children with ADHD. ${ }^{29}$ A significant treatment difference favoring LDX compared to placebo was observed on the ADHD Rating Scale Version IV (ADHD-RS-IV), Conners' Parent Rating Scale-Revised Short Version, and CGI-Improvement with all doses of LDX. The most improvement in the mean ADHD-RS-IV scores and in Connor's Parent Rating Scale-Revised Short Version was observed in patients who received LDX $70 \mathrm{mg}$. Additionally, the CGI-Improvement scores significantly improved from baseline to treatment endpoint for all LDX doses compared with placebo.

Lopez et al, in a post hoc analysis, noted improvements at all three assessment times on the Connor's Parent Rating 


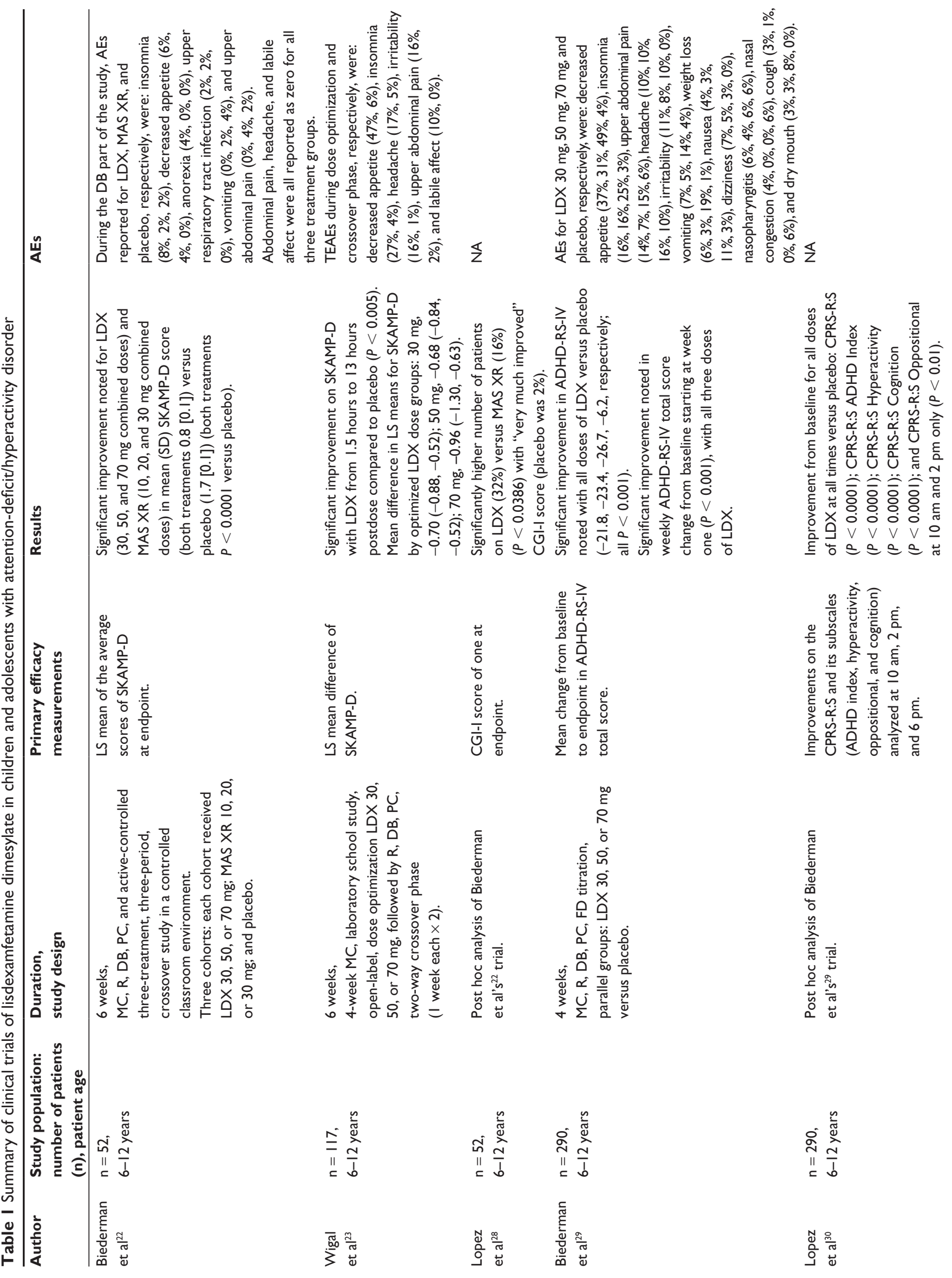



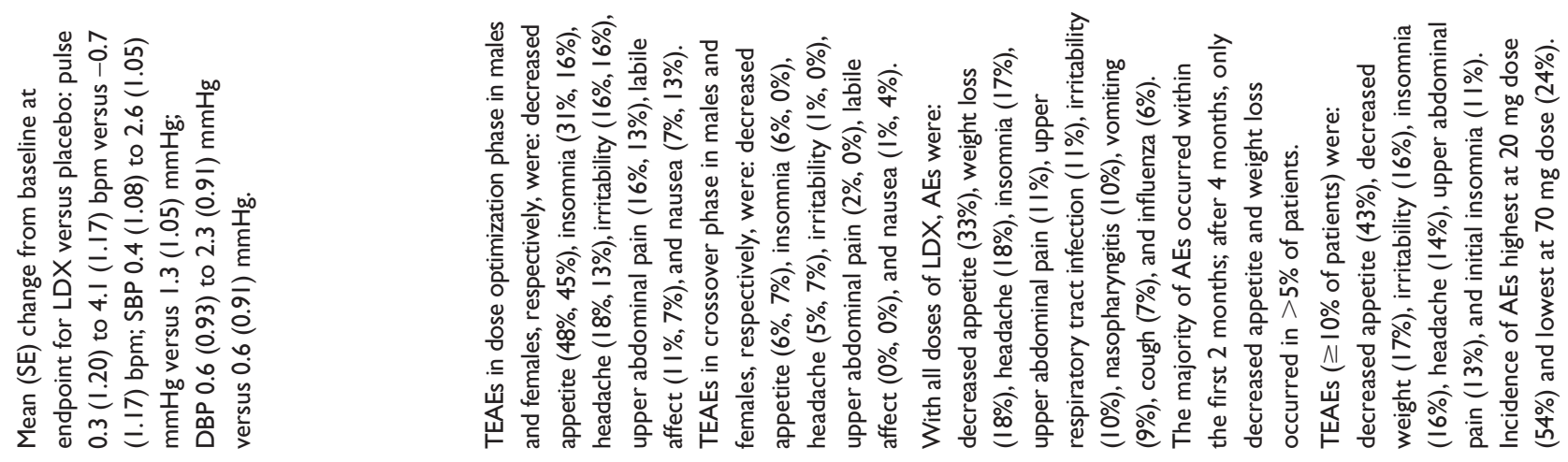
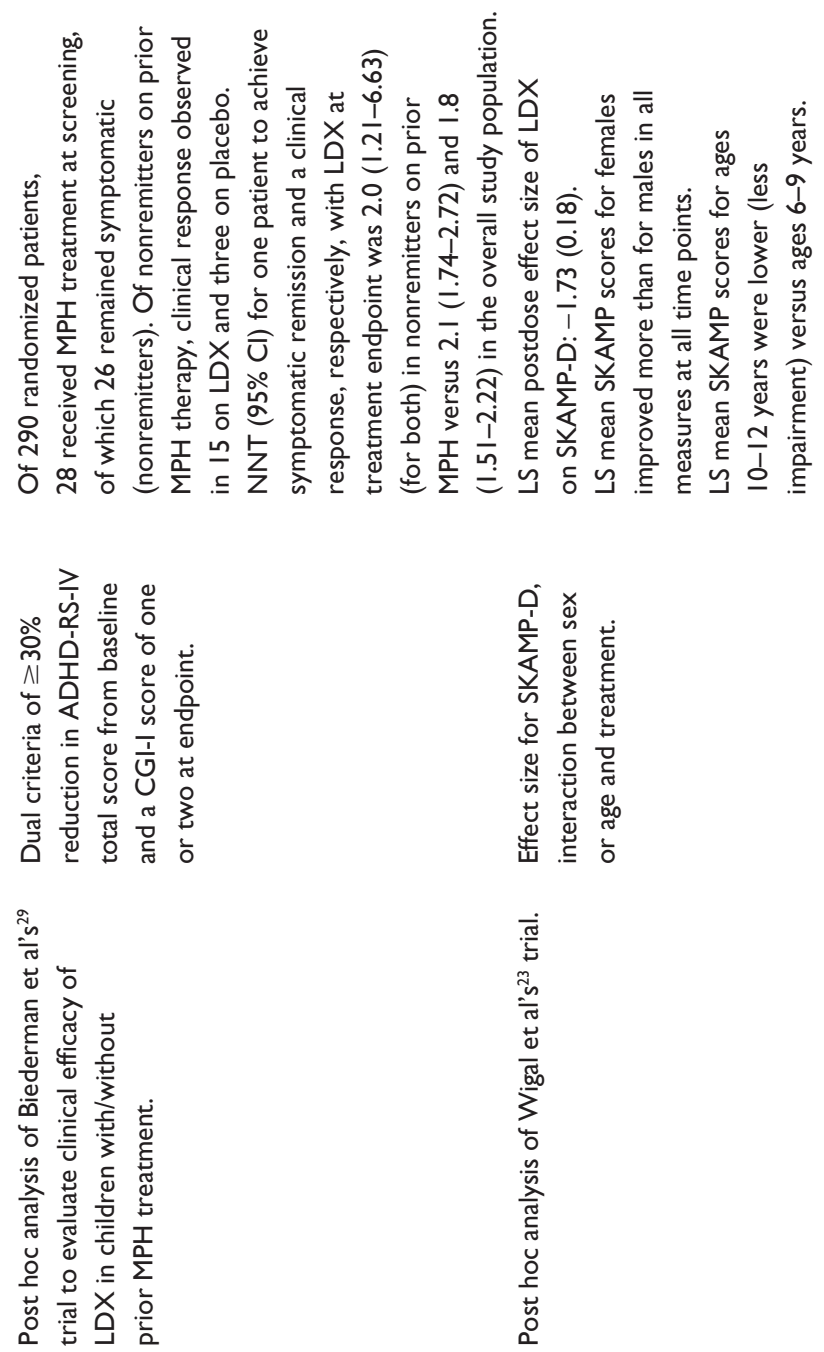

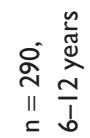

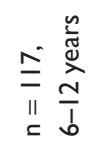

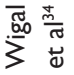

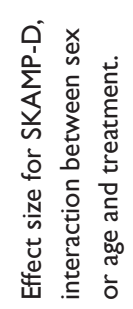

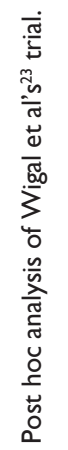

愛

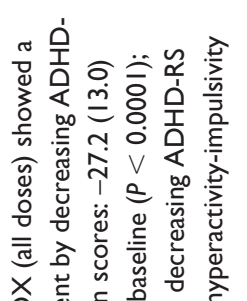

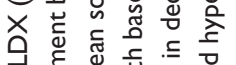

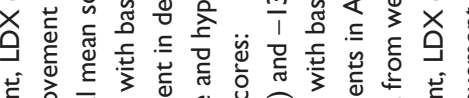

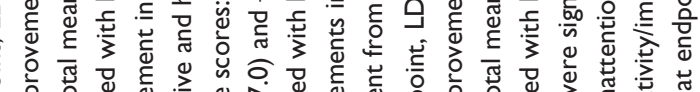

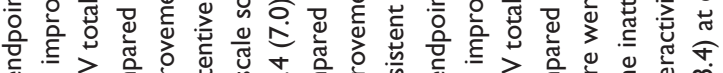

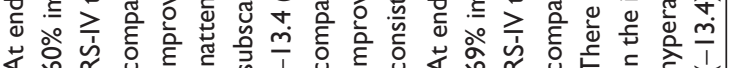
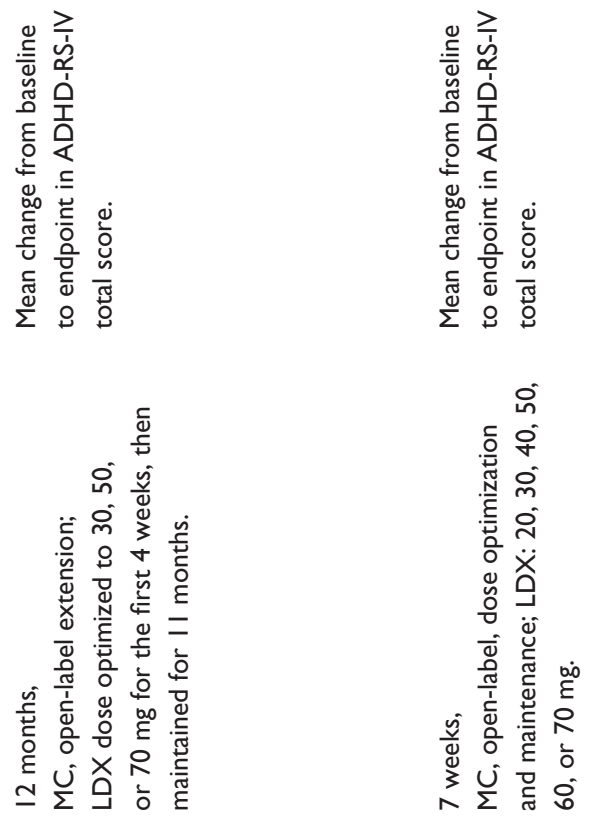

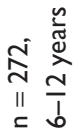

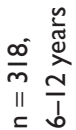

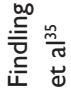

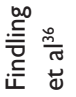




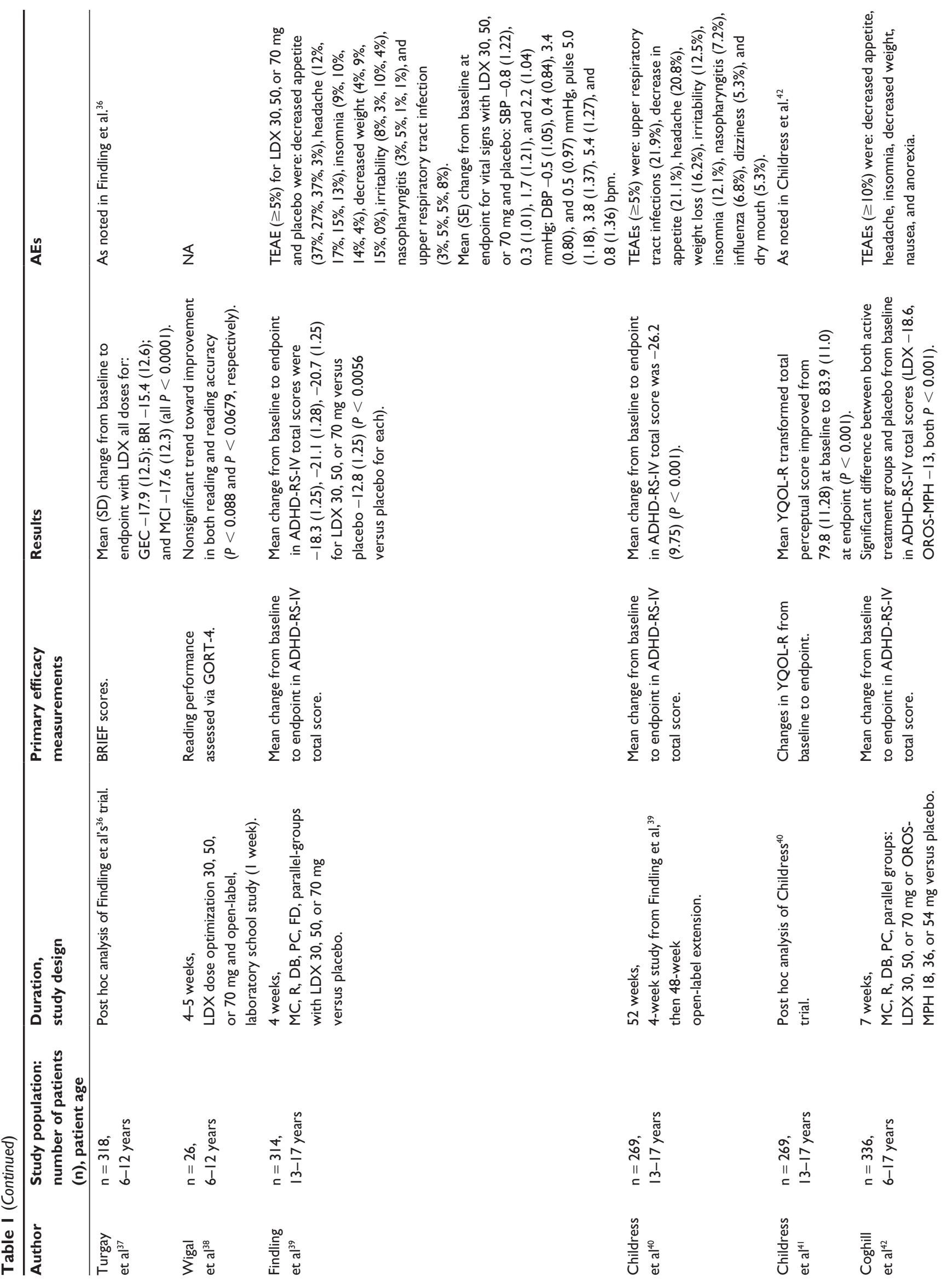



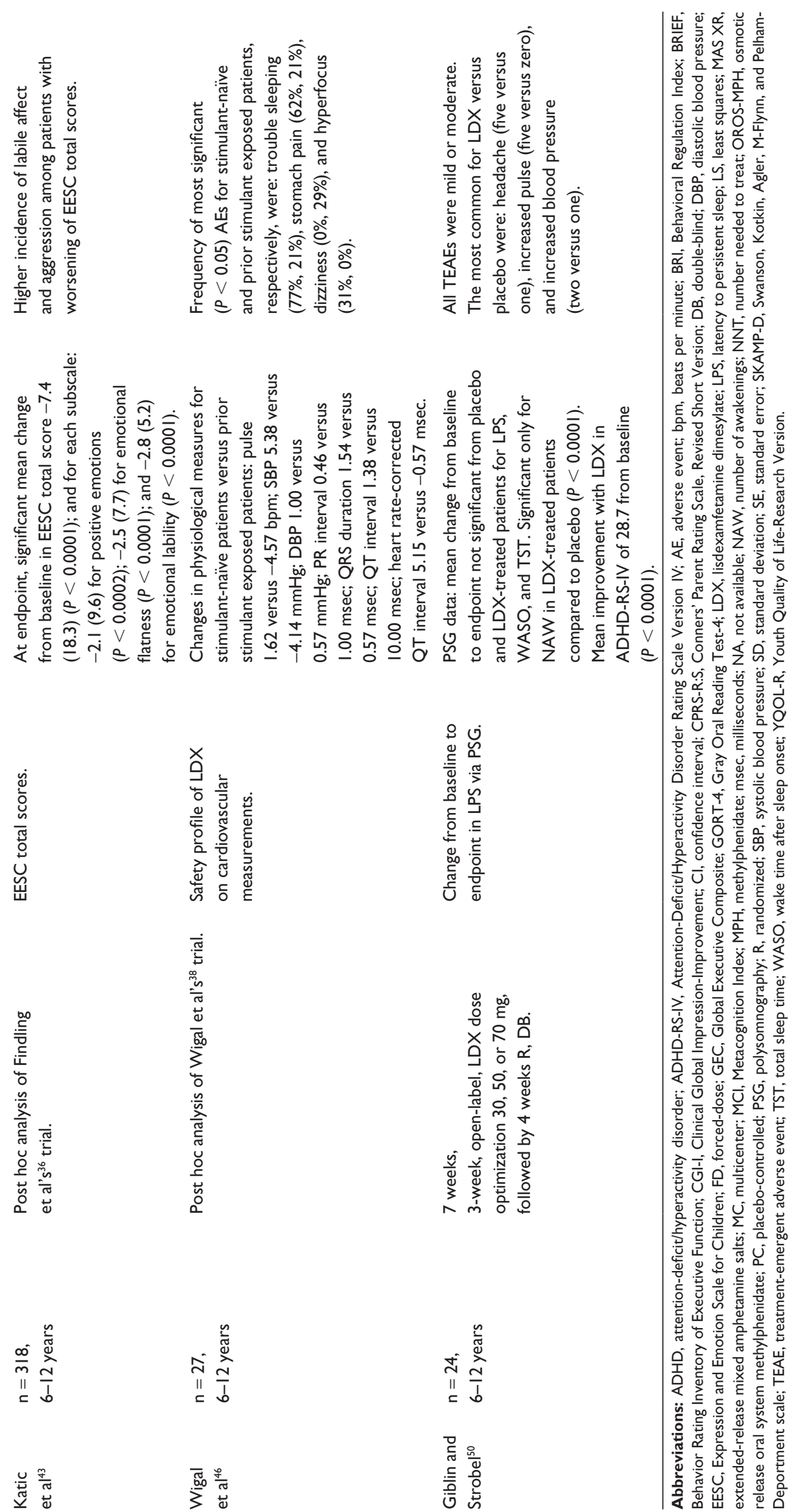

\begin{tabular}{|c|c|c|c|}
\hline 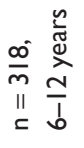 & 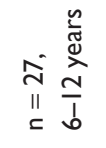 & 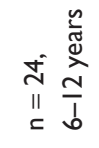 & 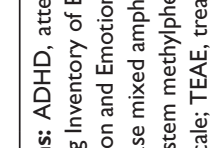 \\
\hline 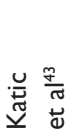 & 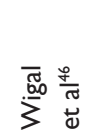 & 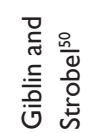 & 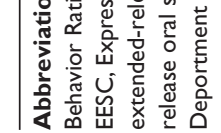 \\
\hline
\end{tabular}

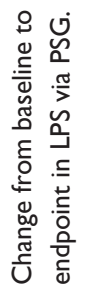
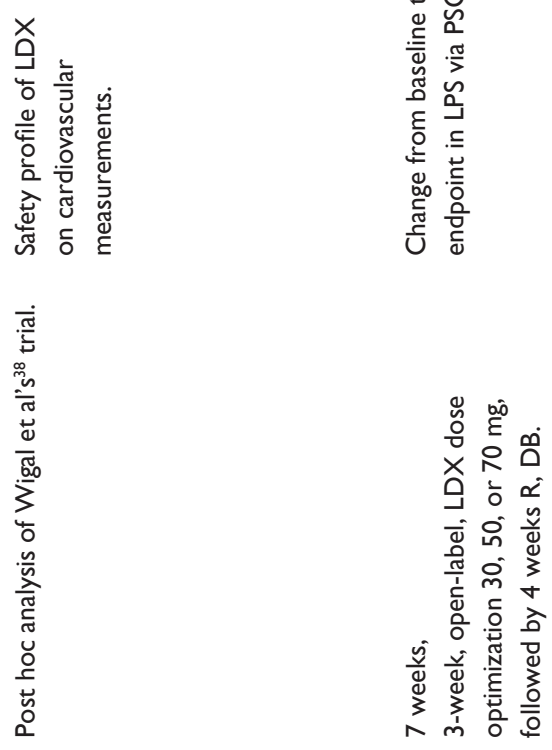

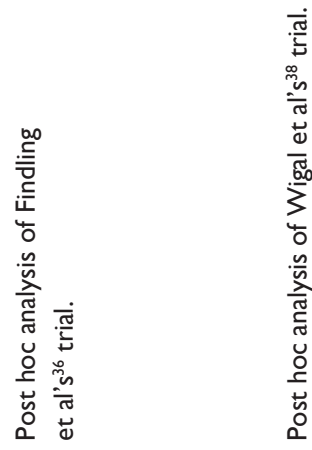

उ 
Scale-Revised Short Version total score and subscales of AHDH Index, Hyperactivity, and Cognition, regardless of the patient's baseline disease severity. ${ }^{30}$ Another post hoc analysis by Jain et al evaluated the clinical efficacy of LDX in children with and without prior methylphenidate treatment at screening. ${ }^{31}$ They found that children with significant clinical ADHD symptoms - despite prior treatment with methylphenidate - improved with LDX treatment, and efficacy outcomes were similar to the results of the overall study population, regardless of the LDX dose utilized.

In Biederman et al's Phase III study, the effect size of LDX treatment was reported at 30,50 , and $70 \mathrm{mg}$ to be $1.21,1.34$, and 1.60 , respectively. ${ }^{29}$ A meta-analysis of stimulants used in the treatment of ADHD in children noted that the largest effect size was observed with LDX treatment. ${ }^{32}$ This high LDX effect size reflected greater efficacy of amphetamine products compared to methylphenidate products, which could not be attributed to measurement artifacts. ${ }^{33}$ However, the results are based on one pediatric clinical trial and the findings did not generalize to adults. Thus, a replication study is needed in children and adolescents before concluding the superiority of LDX over other stimulants.

In a simulated classroom setting involving 117 children with ADHD, Wigal et al conducted a 4-week open-label, dose optimization study of $\operatorname{LDX}(30,50,70 \mathrm{mg} /$ day $)$ followed by a 2-week randomized, placebo-controlled, two-way crossover phase. ${ }^{23}$ Changes from baseline SKAMP-Deportment, SKAMP-Attention, and Permanent Product Measure of Performance scores up to 13 hours postdose were significantly higher in children treated with LDX compared to placebo. ADHD-RS-IV total scores and subscale scores improved from baseline for all LDX doses during the 4-week openlabel phase, and during the 2 weeks of the crossover period. All patients had a CGI-Improvement rating of "very much improved" or "much improved" at the end of the 4 weeks, and $82.3 \%$ of patients had such scores for the crossover period. These continued improvements throughout the day reinforce the benefits derived from the extended duration of action observed with LDX treatment from the previous studies. Post hoc analysis of the above study assessed interaction between sex or age and treatment, and assessed effect sizes for SKAMP. Although both females and males demonstrated improvement on all assessments at postdose time points, females and children between the ages of 10-12 years were noted to have less impairment in SKAMP ratings. ${ }^{34}$

A long-term trial by Findling et al assessed the safety and efficacy of LDX treatment over a 12-month period in 272 children. ${ }^{35}$ LDX was titrated from $30 \mathrm{mg}$ to $70 \mathrm{mg}$ over a period of 4 weeks, and patients continued open-label LDX treatment for an additional 11 months. Clinician-rated ADHD-RS-IV scores improved by a mean of 27.2 points, and improvements occurred during each of the first 4 weeks and were maintained during the study period. More than $80 \%$ of patients were rated as improved at trial endpoint, and more than $95 \%$ of patients had a CGI-Improvement score rated as "very much improved" or "much improved" after completing 12 months of treatment.

Another prospective open-label LDX dose optimization trial was conducted by Findling et al in 318 children. ${ }^{36}$ In this 7-week study evaluating LDX 20, 30, 40, 50, 60, or $70 \mathrm{mg}$, a $69 \%$ average relative improvement from baseline to endpoint in the ADHD-RS-IV total score was observed. Approximately $89 \%$ of patients at endpoint were classified as CGI-Improvement "very much improved" or "much improved," and on the parent-rated Parental Global Assessment scale, $85 \%$ were rated as improved. Additionally, $76 \%$ of parents reported they were "very satisfied" with DX treatment and $87 \%$ stated they would "absolutely" or "probably" continue using LDX as treatment. In a post hoc analysis, efficacy was assessed with the Behavior Rating Inventory of Executive Function - a validated instrument that measures components of executive functioning in children 5-18 years of age. Two indices were used: the Behavioral Regulation Index (inhibit, shift, and emotional control) and Metacognition Index (initiate, working memory, plan/ organize, organization of materials, and monitor); these two indices together comprise a Global Executive Composite score. Significant improvements in the Behavior Rating Inventory of Executive Function indices for all dosages of LDX were demonstrated without regards to ADHD subtype, comorbid psychiatric symptoms, sex, or baseline executive function impairment category. ${ }^{37}$

Wigal et al assessed effects of LDX on reading performance in 26 children with ADHD in a modified laboratory school study with an open-label, dose-optimization phase of a daily dose of 30, 50, or $70 \mathrm{mg}$ LDX. ${ }^{38}$ Following 4-5 weeks of optimal dose titration, the Gray Oral Reading Test-4 - a measure of rate, accuracy, and comprehension - was administered at baseline and at 3-4 hours postdose. At study endpoint, LDX significantly reduced symptoms of ADHD from baseline, as evidenced by ADHD-RS-IV subtypes of hyperactivity/ impulsivity and inattention (both $P<0.0001$ ). No differences were noted for reading accuracy or comprehension. However, reading rate improved, especially among children with higher verbal fluid reasoning without additional symptomatology of neurodevelopmental delay. 


\section{Adolescents}

In late 2010, the FDA approved LDX for use in adolescents based on results from a double-blind, placebo-controlled, 4-week, forced-dose, Phase III trial. ${ }^{39}$ This study by Findling et al was conducted in 45 United States sites in 309 adolescents (aged 13-17 years) who met Diagnostic and Statistical Manual of Mental Disorders (fourth edition, text revision) criteria ${ }^{27}$ for ADHD with at least moderately symptomatic ADHD (ADHD-RS-IV score of $\geq 28$, baseline mean 37-38.5). All patients randomized to LDX were initiated on $30 \mathrm{mg}$ for the first week of treatment and patients assigned to the $50 \mathrm{mg}$ and $70 \mathrm{mg}$ dose were escalated by $20 \mathrm{mg}$ weekly until they reached their assigned dose. This forced-dose titration of 3 weeks was followed by a 1-week dose maintenance phase. The primary and secondary efficacy measures included the ADHD-RS-IV, CGI-Improvement, and Youth Quality Of Life-Research Version (YQOL-R) scores from baseline to endpoint. At endpoint, changes in ADHD-RS-IV total scores were significantly greater for each LDX dose compared to placebo (all $P<0.0056$ ). CGI-Improvement scores showed that $69.1 \%$ of participants treated with LDX (all doses combined) were rated "very much improved" or "much improved" at endpoint compared to $39.5 \%$ of participants on placebo $(P<0.0001)$. There was no statistically significant improvement in the YQOL-R scores at endpoint for LDX compared to placebo.

Patients who participated in the above study were eligible to enroll in a 48 -week maintenance phase, open-label extension of the original 4-week study to further assess the long-term safety and efficacy of LDX. ${ }^{40}$ Patients were seen once monthly and optimized doses were either continued or titrated upwards or downwards to a maximum of $70 \mathrm{mg} /$ day. The mean (standard deviation) ADHD-RS-IV total score at baseline was 38 (7), and the mean change from baseline to endpoint was $-26.2(9.75)(P<0.001)$. At all follow-up visits, significant changes in subscale scores of inattention and hyperactivity/impulsivity were observed: change score of -15.1 (6.05) with a baseline of 22.6 (3.35) and change score of -11.1 (5.89) with a baseline of 15.4 (6.9), respectively $(P<0.001)$. Improvements in GCI-Improvement scores of "very much improved" or "much improved" were noted in $91.2 \%$ of patients at week 4 (dose optimization phase) and in $87.2 \%$ at maintenance phase endpoint. Childress et al also evaluated the quality of life in this long-term open-label trial of LDX: the YQOL-R transformed total perceptual score was 79.8 (11.3) at baseline, $84.6(9.4)$ at week 28, and 83.9 (11.0) at endpoint (all $P<0.001) .{ }^{41}$ Likewise, the YQOL-R domain scores for self, relationship, and environment all improved significantly from baseline to week 28 (all $P<0.001$ ) and to endpoint (all $P<0.001$ ); the general domain improved from baseline to endpoint $(P<0.027)$. Patients with poor participant-perceived scores at baseline showed improvement at endpoint on YQOL-R domain scores (ranging 9.8-17.6) compared to those without poor baseline scores (ranging 0.4-5.1). Likewise, the change in score from baseline for the YQOL-R transformed total perceptual score was 12.5 and 2.9 , respectively, in the same patient subgroups.

A recent Phase III study conducted at 48 sites in Europe enrolled 336 patients (aged 6-17 years) with at least moderate symptoms, defined as a baseline ADHD-RS-IV total score of $\geq 28$ (mean 49). ${ }^{42}$ Patients were randomized to LDX, osmotic release oral system methylphenidate (OROS-MPH), or placebo over a period of 7 weeks. Doses of LDX and OROS-MPH were optimized to 30,50 , or $70 \mathrm{mg}$ /day and 18,36 , or $54 \mathrm{mg} /$ day, respectively. The primary comparison was LDX compared to placebo with no formal comparisons intended between LDX and ORSO-MPH. From baseline to endpoint, significant differences between both active treatment groups and placebo in ADHD-RS-IV total scores were observed $(P<0.001)$. At study endpoint, improvements in CGI-Improvement scores were noted in $78 \%$ of patients in the LDX group, $61 \%$ in the OROS-MPH group, and $14 \%$ in the placebo group. The effect size for LDX was reported at 1.80 compared with 1.26 for OROS-MPH.

\section{Safety data}

Since LDX is a prodrug ultimately converted to d-amphetamine, the expected adverse effect profile of LDX is similar to that of other amphetamine products. LDX was well tolerated in all the clinical trials described above with similar incidence of adverse effects noted in the short-term trials and the longterm open-label trials. Most treatment-emergent adverse events were of mild-to-moderate intensity for all doses of LDX, with no reports of death. The majority of adverse events were noted to occur during the dose titration and dose optimization phases, and most adverse events declined over time (Table 1).

In the Phase II analog classroom study in children aged 6-12 years, the adverse events were consistent with those observed in other stimulants, including MAS XR. ${ }^{22}$ In this study, decreased appetite was noted in both treatment groups (LDX 6\%, MAS XR 4\%), and anorexia occurred only in the LDX group (4\%). A small but significant increase in diastolic blood pressure and pulse were noted among patients treated with LDX (4.6 mmHg and $6.7 \mathrm{bpm}$ ) and MAS XR (2.7 $\mathrm{mmHg}$ and $5.3 \mathrm{bpm}$ ) compared to placebo 2.5 hours 
following stimulant administration. Other assessments, including systolic blood pressure, heart rate-corrected QT intervals, and electrocardiogram (ECG) parameters, did not differ between treatment and placebo groups. ${ }^{22}$

The incidence of adverse events with LDX reported in the three Phase III trials and one open-label short-term study in children aged 6-12 years that were significantly greater than that reported in the placebo group included: appetite suppression $(33 \%-47 \%)$, insomnia $(17 \%-27 \%)$, headache (12\%-18\%), upper abdominal pain $(11 \%-15 \%)$, irritability $(10 \%-16 \%)$, and weight loss $(9 \%-18 \%))^{23,29,35,36}$ No clinically significant changes were observed in laboratory values or physical exams. Small mean increases in blood pressure and pulse and small reductions in weight were observed in the pediatric studies, and were consistent with the known effects of stimulant use.

The occurrence of insomnia and vomiting from the open-label, long-term study in children was noted to be dosedependent. At doses of 30, 50, or $70 \mathrm{mg}$, the occurrence of insomnia and vomiting were $4 \%$ and $3 \%, 9 \%$ and $4 \%$, and $17 \%$ and $6 \%$, respectively. No patient had a QT, Fridericia's heart rate-corrected QT, or Bazett's heart rate-corrected QT interval of $\geq 500$ milliseconds at any treatment visit, and no changes in ECG measurements were determined to be clinically meaningful. Mean (standard deviation) changes from baseline in vitals were 0.7 (10) $\mathrm{mmHg}$ for systolic blood pressure, $0.6(8.3) \mathrm{mmHg}$ for diastolic blood pressure, and 1.4 (13.7) bpm for pulse. In the second 8-week treatment period of the study, decrease only in weight and appetite occurred in $\geq 5 \%$ of patients, indicating that tolerability to LDX improved over time..$^{35}$

In the dose optimization phase of the 13-hour laboratory school study, the most commonly observed adverse events in males were upper abdominal pain, headache, labile affect, and insomnia; in females it was nausea and decreased weight. During the crossover phase for those receiving LDX, males experienced more upper abdominal pain and insomnia, and females reported more nausea and headache. ${ }^{34}$

A post hoc analysis of the 7-week, open-label, dose optimization study with LDX aimed to describe the reliability and clinical relevance of change in emotional expression using the Expression and Emotion Scale for Children (EESC), a parent-rated report that evaluates both positive and negative aspects of emotional expression..$^{43}$ At endpoint, improvement was noted from baseline in EESC total score. Additionally, a significant decrease from baseline for each EESC subscale (emotional flatness, positive emotions, and emotional lability) score was noted in patients at their last study visit, suggesting there was no mean worsening of emotional expression scores. Findings from this study are limited due to a lack of a placebo group for comparison and normative data for the EESC; however, results may suggest that LDX does not adversely affect emotional expression overall for many participants and, for some, improvement from baseline was achieved.

The most frequently reported adverse events in the 4-week, forced-dose titration study involving adolescents aged 13-17 years taking LDX were decreased appetite, headache, insomnia, weight loss, and irritability. ${ }^{39}$ Small mean increases in pulse and blood pressure from baseline to endpoint were observed in LDX-treated patients compared to placebo. At endpoint, a pulse of $\geq 100 \mathrm{bpm}$ was noted in $5 \%$, $1 \%$, and $3 \%$ of patients treated with LDX 30,50 , or $70 \mathrm{mg}$, respectively, compared to $1 \%$ on placebo. Systolic blood pressure of $\geq 120 \mathrm{mmHg}$ was observed in $30 \%, 28 \%$, and $27 \%$ of patients treated with LDX 30, 50, and $70 \mathrm{mg}$, respectively, compared to $25 \%$ on placebo at endpoint. Mean increase in Fridericia's heart rate-corrected interval at endpoint was observed for all three LDX doses (0.2-2.7 milliseconds) compared to placebo (2.8 milliseconds), with no differences across treatment groups. Two participants had postbaseline ECG findings determined to be clinically significant (Fridericia's heart rate-corrected QT interval of 479 milliseconds and 413 milliseconds, respectively), which led to study discontinuation. This differed from the earlier aforementioned pediatric studies where no clinically significant cardiac conduction changes were observed. No new or unexpected safety concerns were observed in laboratory or physical examinations, and overall the treatment-emergent adverse events were consistent with previous LDX studies and previously described effects of amphetamines in children and adults.

The safety analysis from the long-term, open-label extension study in adolescents noted that most treatment-emergent adverse events were mild to moderate in severity and 15 serious adverse events were reported in ten subjects; only syncope (three episodes) was thought to be treatment related. At endpoint, small mean (standard deviation) increases in systolic blood pressure $(2.3[10.53] \mathrm{mmHg})$, diastolic blood pressure (2.5 [8.37]) $\mathrm{mmHg}$, and pulse (6.3 [12.74]) bpm were observed from baseline. No clinically significant ECG changes at endpoint or clinically meaningful trends in laboratory were observed. ${ }^{40}$

In the adolescent European study, treatment-emergent adverse events were reported in $65 \%, 72 \%$, and $57 \%$ of patients in the OROS-MPH, LDX, and placebo groups, respectively. ${ }^{42}$ 
Modest changes in vitals and ECG were observed in patients receiving stimulants, but the ECG changes were deemed not clinically significant. Most common ( $\geq 10 \%)$ treatmentemergent adverse events reported by patients were decreased appetite, headache, insomnia, decreased weight, nausea, and anorexia. Further analysis of the results from this first European, Phase III study of once-daily LDX are expected to be published soon.

The effect of LDX on growth of children has been evaluated by Faraone et al in an exploratory uncontrolled study from previous trials with LDX 30,50 , or $70 \mathrm{mg}$ in 281 children (aged 6-13 years), with longitudinal assessments on height, weight, and body mass index up to 15 months. ${ }^{44}$ At baseline, children with ADHD were taller and heavier than average when compared to norms from the Centers for Disease Control and Prevention, and although children treated with LDX continued to grow in height, the growth delays were largest for weight and body mass index. Body mass index scores decreased significantly from baseline to endpoint $\left(\mathrm{t}_{276}=10.15 ; P<0.0001\right)$. Mean loss was also observed in expected weight $(3.7 \mathrm{~kg})$ and height $(0.9 \mathrm{~cm})$. This study did not separate out the effects of dose and duration; however, study findings did suggest that participants who received prior stimulant therapy had already experienced the bulk of their growth deficit prior to being treated with LDX. The delays were greatest for the heaviest and tallest children, those with the highest cumulative dose exposure, and those who had not received a prior stimulant therapy. The data from this study were similar to the results of the Multimodal Treatment Study of ADHD; ${ }^{45}$ both studies noted that stimulants are associated with growth delays and that these delays decreased over time.

To address the safety profile of LDX based on cardiovascular measurements, Wigal et al conducted a post hoc analysis of the investigation on reading performance in response to LDX in 27 children, of which 14 had prior stimulant exposure and 13 were stimulant naïve. ${ }^{46}$ More cardiovascular effects were measured in stimulant-naïve children than in children who had prior exposure to stimulant therapy. In the stimulant-naïve group, two patients experienced adverse events outside the normal range: one had tachycardia and one had blood pressure in the $\geq 95$ th percentile of normal age range.

A large retrospective cohort study assessed serious cardiovascular events from ADHD medications in over 1.2 million children and young adults aged 2-24 years (mean age at baseline 11 years), with over 2.5 million person-years of follow-up and over 370,000 person-years of current use of
ADHD medications. ${ }^{47}$ Compared with nonusers, the adjusted rate of serious cardiovascular events did not differ significantly among individuals currently using ADHD medications nor among former users. Increased risk was associated with older age, concurrent use of an antipsychotic, a major psychiatric illness, serious cardiovascular condition, and a chronic illness. Although results from this study showed that use of ADHD medications did not increase the risk of serious cardiovascular events, the labeling for LDX - as with other amphetamine products - includes a boxed warning describing sudden cardiac death and serious cardiovascular adverse events with misuse of the medication. In 2008, the American Academy of Pediatrics issued a recommendation that children with ADHD be screened for cardiac problems prior to initiating therapy with stimulants. The recommendations include a physical exam and an evaluation for presence of an abnormal heart murmur, cardiovascular abnormalities, and Marfan syndrome. Since some cardiac conditions may not be detectable on routine physical exams, an ECG may be used. ${ }^{48}$ Later that year, a joint statement by the American Academy of Pediatrics and the American Heart Association stated that ECGs are not mandatory and that they may be performed at the discretion of the physician. ${ }^{49}$

Giblin and Strobel assessed the mean change from baseline to week 7 on objective sleep measures via polysomnography in placebo- and LDX-treated participants. ${ }^{50}$ There were no statistically significant differences noted in both groups regarding latency to persistent sleep, wake time after sleep onset, or total sleep time. Only the number of awakenings after sleep onset significantly decreased in the LDX-treated group relative to baseline $(P<0.0001)$, possibly indicative of a more consolidated sleep. Parents/guardians in this study had extensive sleep hygiene counseling at each visit and appropriate sleep schedules were set for each study participant, which may have also contributed to the lack of sleep disturbances observed with LDX in this study. In comparison, earlier studies have reported an incidence of insomnia of $27 \%$ in children treated with LDX. ${ }^{23}$ The sample in Giblin and Strobel's study was small $(\mathrm{n}=24)$ and the multifarious nature of findings warrant that these conclusions be interpreted cautiously as more studies need to be undertaken in larger samples in children with ADHD. Additionally, the majority of children in this study had received earlier stimulant therapy for ADHD, which may have made them less prone to experience insomnia. Wigal et al had also noted that in stimulant-naïve participants, $77 \%$ experienced "trouble sleeping" while $21 \%$ with prior stimulant exposure reported this effect. ${ }^{46}$ 
Postmarketing data from a poison control review of LDX that involved five poison centers covering eight states noted the most common adverse events reported by the examining health care provider included tachycardia (73\%), agitation (53\%), dystonia (47\%), insomnia (20\%), hallucinations (20\%), chest pain (13\%), fasciculation (20\%), and vomiting (13\%). Additionally, abdominal pain, tremor, confusion, and seizures were each reported at $7 \% .{ }^{51}$

A case of generalized alopecia has been described in a 5 -year-old female following 5 days of treatment with LDX $30 \mathrm{mg} .{ }^{52}$ Two days following discontinuation of LDX, the alopecia was less marked. Eosinophilic hepatitis necessitating hospitalization in a 14-year-old male, whose only prescribed medication had been LDX $30 \mathrm{mg}$ for the previous 5 months, resolved completely within 2 months following LDX discontinuation..$^{53}$ In both the above reports, the Naranjo Scale yielded a score of seven, indicating probable medicationrelated event with LDX.

Goodman et al assessed the safety profile of LDX across age groups from three studies in children, adolescents, and adults. ${ }^{54}$ Common adverse events observed with LDX (all doses) versus placebo are listed in Table 2. The adverse events were similar across age groups, and results were consistent with the safety profile of long-acting stimulants. In children, the incidence of upper abdominal pain and decreased appetite was higher than in adults, while dry mouth and headache were higher in adults than in children.

\section{Clinical applications and place in therapy}

Since ADHD is associated with cognitive, social, and academic impairments, and the pervasive impact of this neurological developmental disorder extends beyond the classroom or school day, the use of a long-acting stimulant may be preferred. Although long-acting stimulants have similar duration of action, as well as safety and tolerability profiles, there are some subtle differences that may aid in the selection of one agent over another. Currently available formulations of long-acting stimulants rely on breakdown of bead coatings to delay drug delivery and may be susceptible to variations in time to onset and duration of action due to interpatient variations in gastric $\mathrm{pH}$. The conversion of LDX to $\mathrm{d}$-amphetamine is not affected by gastrointestinal $\mathrm{pH}$ or gastrointestinal transit times, and the drug has low patientto-patient pharmacokinetic variability, indicating consistent delivery of d-amphetamine. A recent review of long-acting ADHD medications noted considerable interindividual variation with the once-daily formulations of methylphenidate and MAS XR. ${ }^{17}$ LDX may be mixed in a liquid for ease of administration in patients who may not be taught to swallow pills. This cannot be done with the sustained release preparations

Table 2 Summary of the safety profile of lisdexamfetamine dimesylate from three randomized, 4-week, double-blind, placebocontrolled, forced-dose titration studies in children, adolescents, and adults

\begin{tabular}{|c|c|c|c|}
\hline Measure & $\begin{array}{l}\text { Children } \\
\text { (6-12 years) }\end{array}$ & $\begin{array}{l}\text { Adolescents } \\
\text { (13-17 years) }\end{array}$ & $\begin{array}{l}\text { Adults } \\
\text { (18-55 years) }\end{array}$ \\
\hline \multicolumn{4}{|l|}{ Common $\mathrm{AE}(>10 \%)$} \\
\hline \multicolumn{4}{|l|}{ LDX versus placebo } \\
\hline Upper abdominal pain & $11.9 \%$ versus $5.6 \%$ & $0.9 \%$ versus $3.9 \%$ & $2.5 \%$ versus $1.6 \%$ \\
\hline Decreased appetite & $39 \%$ versus $4.2 \%$ & $33.9 \%$ versus $2.6 \%$ & $26.5 \%$ versus $1.6 \%$ \\
\hline Dry mouth & $4.6 \%$ versus $0 \%$ & $4.3 \%$ versus $1.3 \%$ & $25.7 \%$ versus $3.2 \%$ \\
\hline Headache & $11.9 \%$ versus $9.7 \%$ & $14.6 \%$ versus $13 \%$ & $20.7 \%$ versus $12.9 \%$ \\
\hline Insomnia & $18.8 \%$ versus $2.8 \%$ & $11.2 \%$ versus $3.9 \%$ & $19.3 \%$ versus $4.8 \%$ \\
\hline \multicolumn{4}{|l|}{ Changes in vitals: least squares mean (SE) } \\
\hline \multicolumn{4}{|c|}{ change from baseline to endpoint for LDX $30 \mathrm{mg}$, } \\
\hline \multicolumn{4}{|l|}{$50 \mathrm{mg}, 70 \mathrm{mg}$, and placebo, respectively } \\
\hline $\mathrm{SBP}, \mathrm{mmHg}$ & $\begin{array}{l}0.4(1.08), 1.8(1.06) \\
2.6(1.05), 1.3(1.05)\end{array}$ & $\begin{array}{l}-0.8(\mathrm{I} .22), 0.3(\mathrm{I} .0 \mathrm{I}) \\
\mathrm{I} .7(\mathrm{I} .2 \mathrm{I}), 2.2(\mathrm{I} .04)\end{array}$ & $\begin{array}{l}0.8(0.77), 0.3(0.77) \\
1.3(0.75),-0.6(1.05)\end{array}$ \\
\hline $\mathrm{DBP}, \mathrm{mmHg}$ & $\begin{array}{l}0.6(0.93), 1.9(0.92) \\
2.3(0.91), 0.6(0.91)\end{array}$ & $\begin{array}{l}-0.5(1.05), 0.4(0.84) \\
3.4(0.80), 0.5(0.97)\end{array}$ & $\begin{array}{l}0.8(0.61), \text { I.I }(0.60) \\
\text { I.6 (0.60), I.I }(0.83)\end{array}$ \\
\hline Pulse, bpm & $\begin{array}{l}0.3(1.20), 2.0(1.18) \\
4.1(1.17),-0.7(1.17)\end{array}$ & $\begin{array}{l}5.0(1.18), 3.8(1.37) \\
5.4(1.27), 0.8(1.36)\end{array}$ & $\begin{array}{l}2.8(0.83), 4.2(0.83) \\
5.2(0.82),-0.0(1.14)\end{array}$ \\
\hline $\begin{array}{l}\text { Mean (SD) changes in body weight (Ib) } \\
\text { with LDX }\end{array}$ & $-2.5(3.37)$ & $-4.8(3.48)$ & $-4.3(4.49)$ \\
\hline
\end{tabular}

Note: Data drawn from Goodman DW, Scheckner B, Dirks B, et al. Safety profile of lisdexamfetamine dimesylate in clinical trials in children, adolescents, and adults with attention-deficit/hyperactivity disorder. Proceedings of the 163rd Annual Meeting of the American Psychiatric Association; May 22-26, 201 I; New Orleans, LA. ${ }^{54}$

Abbreviations: AE, adverse event; DBP, diastolic blood pressure; LDX, lisdexamfetamine dimesylate; SBP, systolic blood pressure; SD, standard deviation; SE, standard error. 
since they must be swallowed whole, and not be crushed, in order to preserve their long-acting effects. Studies note that the appetite suppression observed with LDX and other amphetamines may be more pronounced than that observed with methylphenidate products with similar duration of action. ${ }^{55}$ LDX demonstrated significant ADHD symptom improvement 1.5-13 hours following oral administration, from morning through homework and family time. ${ }^{23}$

The long duration of effect may be beneficial for adolescents, since their activities may extend beyond the regular school hours and into after-school activities, employment, and other responsibilities. On the other hand, the extended duration of action of this prodrug may be too long for some younger children. Although insomnia may be a concern for some patients due to LDX's long duration of effect, in clinical trials it did not often result in discontinuation of the stimulant.

In addition to the long duration of action noted with LDX, a post hoc analysis by Jain et $\mathrm{al}^{31}$ of the Phase III trial by Biederman et $\mathrm{al}^{29}$ noted that children with significant clinical ADHD symptoms - despite prior treatment with methylphenidate - improved on LDX, regardless of dose, and had similar improvements in their symptoms as the overall study population. In the same Phase III trial, analysis of ADHD-RS-IV scores at treatment endpoint noted the effect size of LDX 30, 50, and $70 \mathrm{mg}$ to be $1.21,1.34$, and 1.60 , respectively. ${ }^{29}$ Faraone and Buitelaar, in a meta-analysis of stimulant medications used in children with ADHD, noted that regarding the number needed to treat $(95 \%$ confidence interval) results, clinicians would need to treat two (1.7-2.2) patients with amphetamine compared with 2.6 (2.4-2.8) with methylphenidate for each positive outcome for total ADHD symptoms. ${ }^{32}$ The meta-analysis also noted that the largest effect size was similar to that observed in Biederman et al's Phase III trial ${ }^{29}$ and the apparent superiority of LDX may be that it reflects greater efficacy of amphetamine compared to nonamphetamine (eg, methylphenidate) products, a finding noted by the meta-analysis and supported by a comparative review by Arnold. ${ }^{56}$ The difference in effect size between amphetamine and nonamphetamine stimulants (eg, methylphenidate) may be due to differences in the molecular mechanisms implicated in facilitating the dopaminergic neurotransmission, and although the pediatric trial by Biederman et $\mathrm{a}^{29}$ suggests that LDX is more efficacious than other psychostimulants, more clinical trials need to be undertaken to see if such findings can be replicated.

Clinicians need to be vigilant when prescribing stimulants as they may be abused or diverted, especially the immediate release formulations that have a quick onset of action. LDX requires oral ingestion to convert it from an inactive form to the active drug, d-amphetamine, thus making it less likely to be susceptible to misuse or abuse by other delivery routes (eg, inhalation, injection), and may also have benefit in a household where abuse or misuse is a concern. Support for the reduced abuse potential with oral and intravenous LDX relative to immediate release d-amphetamine has been described in adult non-ADHD subjects with a history of drug abuse..$^{57,58}$ In those studies, the abuse-related liking scores for oral LDX at a dose of $150 \mathrm{mg}$ (amphetamine base content equivalent to d-amphetamine $60 \mathrm{mg}$ ) were comparable to oral d-amphetamine $40 \mathrm{mg}$ (an amphetamine-based dose equivalent to LDX $100 \mathrm{mg}) .{ }^{57}$ Intravenous d-amphetamine $20 \mathrm{mg}$, but not intravenous LDX $50 \mathrm{mg}$, produced significantly more liking effects compared to placebo. ${ }^{58}$

Poor medication adherence rates among patients with ADHD occur across all ages, and may be more problematic among teenagers. Medication compliance may be affected by patient beliefs about the disorder; side effects of the medication; and, in adolescents, concerns about peer group acceptance and the stigma of the illness, or concerns about taking the medications. One review of prescription claims in children ( $\leq 18$ years) noted mean adherence rates (medication possession ratio $\geq 80 \%$ ) during the school year and during the entire year, respectively, for immediate release stimulants (52.8\% versus $37.2 \%)$, extended release stimulants $(63.7 \%$ versus $52.1 \%$ ), LDX (63.5\% versus $47.6 \%$ ), and nonstimulants (62.9\% versus $52.5 \%$ ). Additionally, mean adherence rates were higher during the school year than the entire year for long-acting agents $(63.4 \%$ versus $53.3 \%)$ than for the shortacting agents $(52.2 \%$ versus $37.2 \%) .{ }^{59}$ Symptoms of ADHD have an additional impact on the adaptive functioning and quality of life. Patrick et al found that adolescents with ADHD had a reported mean YQOL-R transformed total perceptual score of 75.2 compared to 82.2 for controls. ${ }^{60}$ The study by Childress et al noted that the scores at endpoint (83.9) were similar to that of controls, demonstrating the improved quality of life observed with LDX in that patient population. ${ }^{41}$

Antonucci et al assessed parents' perceptions regarding the impact of ADHD and use of LDX in children via surveys ( $\mathrm{n}>11,000)$ in a real-world setting. ${ }^{61}$ Parents reported significant improvements in symptoms that caused substantial interference with school activities, homework, and family and social interactions $(P<0.01)$. Satisfaction with LDX was significantly higher than with their child's previous treatment $(P<0.01)$. On average, tolerability, global improvement, convenience, and satisfaction with LDX were all highly rated and when asked about intention to continue treatment, $83 \%$ responded yes, 14\% maybe, and 3\% no. 
LDX is commercially available in 20, 30, 40, 50, 60, and $70 \mathrm{mg}$ capsules. Doses in children and adolescents should be initiated at $30 \mathrm{mg}$ once daily in the morning, regardless of prior stimulant use. The dose may be increased by $10 \mathrm{mg}$ or $20 \mathrm{mg} /$ day at approximately weekly intervals to a maximum daily dose of $70 \mathrm{mg}$. Patients who have had prior exposure to stimulant therapy may tolerate titration of LDX to higher doses better than stimulant-naïve patients. ${ }^{45}$

\section{Conclusion}

The chronic nature of ADHD extends well beyond childhood and, in adolescents, may lead to a variety of risk-taking behaviors, which can have a significant adverse consequence for development and adult well-being. LDX, a prodrug of d-amphetamine, is a once-daily medication indicated for the treatment of ADHD in children and adolescents. Efficacy results in adolescents were consistent with the earlier findings in children, and the safety profile for LDX is similar to other currently marketed stimulants, with increases in blood pressure and pulse appearing to be dose-related. LDX has reduced toxicity in an overdose, and reduced liability for misuse and abuse. The extended duration of action observed with LDX provides sufficient time to control ADHD symptoms that extend beyond the school day and well into after-school activities, homework, and family time. Until new medications are discovered with a similar efficacy as the currently available stimulants, but with a better tolerated safety profile and reduced risk for abuse, stimulants - like LDX - will remain drugs of choice in managing ADHD.

\section{Disclosure}

The author reports no conflicts of interest in this work.

\section{References}

1. Wolraich ML, Wibbelsman CJ, Brown TE, et al. Attention-deficit/ hyperactivity disorder among adolescents: a review of the diagnosis, treatment, and clinical implications. Pediatrics. 2005;115(6): 1734-1746.

2. Subcommittee on Attention-Deficit/Hyperactivity Disorder, Steering Committee on Quality Improvement and Management. ADHD: clinical practice guideline for the diagnosis, evaluation, and treatment of attention-deficit/hyperactivity disorder in children and adolescents. Pediatrics. 2011;128(5):1007-1022.

3. Froehlich TE, Lanphear BP, Epstein JN, Barbaresi WJ, Katusic SK, Kahn RS. Prevalence, recognition, and treatment of attention-deficit/ hyperactivity disorder in a national sample of US children. Arch Pediatr Adolesc Med. 2007;161(9):857-864.

4. Green JG, Avenevoli S, Finkelman M, et al. Attention deficit hyperactivity disorder: concordance of the adolescent version of the Composite International Diagnostic Interview Version 3.0 (CIDI) with the K-SADS in the US National Comorbidity Survey Replication Adolescent (NCS-A) supplement. Int J Methods Psychiatr Res. 2010;19(1):34-49.
5. Doyle AE. Executive functions in attention-deficit/hyperactivity disorder. J Clin Psychiatry. 2006;67 Suppl 8:21-26.

6. Canadian Attention Deficit Hyperactivity Disorder Resource Alliance (CADDRA). Canadian ADHD practice guidelines. Updated Oct 2011. Available from: http://www.caddra.ca/cms4/pdfs/caddra Guidelines2011.pdf. Accessed January 2, 2012.

7. Pliszka S; AACAP Work Group on Quality Issues. Practice parameter for the assessment and treatment of children and adolescents with attention-deficit/hyperactivity disorder. J Am Acad Child Adolesc Psychiatry. 2007;46(7):894-921.

8. Kutcher S, Aman M, Brooks SJ, et al. International consensus statement on attention-deficit/hyperactivity disorder (ADHD) and disruptive behavior disorders (DBDs): clinical implications and treatment practice suggestions. Eur Neuropsychopharmacol. 2004; 14(1):11-28.

9. AAP.org [homepage on the Internet]. Implementing the key action statements: an algorithm and explanation for process of care for the evaluation, diagnosis, treatment, and monitoring of ADHD in children and adolescents. 2011. Available from: http://pediatrics.aappublications. org/content/supp1/2011/10/11/peds.2011-2654.DC1/zpe611117822p. pdf. Accessed January 3, 2012.

10. Adler LA, Mattingly GW, Montano CB, Newcorn JH. Optimizing clinical outcomes across domains of life in adolescents and adults with ADHD. J Clin Psychiatry. 2011;72(7):1008-1014.

11. Wilens TE, Spencer TJ. Understanding attention-deficit/hyperactivity disorder from childhood to adulthood. Postgrad Med. 2010;122(5): 97-109.

12. Bussing R, Mason DM, Bell L, Porter P, Garvan C. Adolescent outcomes of childhood attention-deficit/hyperactivity disorder in a diverse community sample. JAm Acad Child Adolesc Psychiatry. 2010; 49(6):595-605.

13. Findling RL. Evolution of the treatment of attention-deficit/hyperactivity disorder in children: a review. Clin Ther. 2008;30(5):942-957.

14. Connor DF, Steingard RJ. New formulations of stimulants for attentiondeficit hyperactivity disorder: therapeutic potential. CNS Drugs. 2004; 18(14):1011-1030.

15. Brams M, Moon E, Pucci M, Lopez FA. Duration of effect of oral longacting stimulant medications for ADHD throughout the day. Curr Med Res Opin. 2010;26(8):1809-1825.

16. Brams M, Mao AR, Doyle RL. Onset of efficacy of long-acting psychostimulants in pediatric attention-deficit/hyperactivity disorder. Postgrad Med. 2008;120(3):69-88.

17. Banaschewski T, Coghill D, Santosh P, et al. Long-acting medications for the hyperkinetic disorders. A systematic review and European treatment guideline. Eur Child Adolesc Psychiatry. 2006;15(8): 476-495.

18. Pennick M. Absorption of lisdexamfetamine dimesylate and its enzymatic conversion to d-amphetamine. Neuropsychiatr Dis Treat. 2010;6:317-327.

19. Ermer J, Homolka R, Martin P, Buckwalter M, Purkayastha J, Roesch B. Lisdexamfetamine dimesylate: linear dose-proportionality, low intersubject and intrasubject variability, and safety in an open-label single-dose pharmacokinetic study in healthy adult volunteers. $J$ Clin Pharmacol. 2010;50(9):1001-1010.

20. Najib J. The efficacy and safety profile of lisdexamfetamine dimesylate, a prodrug of d-amphetamine, for the treatment of attention-deficit/hyperactivity disorder in children and adults. Clin Ther. 2009;31(1): 142-176.

21. Boellner SW, Stark JG, Krishnan S, Zhang Y. Pharmacokinetics of lisdexamfetamine dimesylate and its active metabolite, d-amphetamine, with increasing oral doses of lisdexamfetamine dimesylate in children with attention-deficit/hyperactivity disorder: a single-dose, randomized, open-label, crossover study. Clin Ther. 2010;32(2):252-264.

22. Biederman J, Boellner SW, Childress A, Lopez FA, Krishnan S, Zhang Y. Lisdexamfetamine dimesylate and mixed amphetamine salts extended-release in children with ADHD: a double-blind, placebo-controlled, crossover analog classroom study. Biol Psychiatry. 2007;62(9):970-976. 
23. Wigal SB, Kollins SH, Childress AC, Squires L; 311 Study Group. A 13-hour laboratory school study of lisdexamfetamine dimesylate in school-aged children with attention-deficit/hyperactivity disorder. Child Adolesc Psychiatry Ment Health. 2009;3(1):17-30.

24. Krishnan S, Moncrief S. An evaluation of the cytochrome p450 inhibition potential of lisdexamfetamine in human liver microsomes. Drug Metab Dispos. 2007;35(1):180-184.

25. Ermer JC, Adeyi BA, Pucci ML. Pharmacokinetic variability of long-acting stimulants in the treatment of children and adults with attention-deficit hyperactivity disorder. CNS Drugs. 2010;24(12): 1009-1025.

26. Adderall $\mathrm{XR}^{\circledR}$ [package insert]. Wayne, PA: Shire US Inc; 2011.

27. American Psychiatric Association. Attention-deficit and disruptive behavior disorders. In: Diagnostic and Statistical Manual of Mental Disorders DSM-IV-TR. 4th ed. Text Revision. Washington, DC: American Psychiatric Association; 2000:85-103.

28. Lopez FA, Scheckner B, Childress AC. Physician perception of clinical improvement in children with attention-deficit/hyperactivity disorder: a post hoc comparison of lisdexamfetamine dimesylate and mixed amphetamine salts extended release in a crossover analog classroom study. Neuropsychiatr Dis Treat. 2011;7:267-273.

29. Biederman J, Krishnan S, Zhang Y, McGough JJ, Findling RL. Efficacy and tolerability of lisdexamfetamine dimesylate (NRP-104) in children with attention-deficit/hyperactivity disorder: a phase III, multicenter randomized, double-blind, forced-dose, parallel-group study. Clin Ther. 2007;29(3):450-463.

30. Lopez FA, Ginsberg LD, Arnold V. Effect of lisdexamfetamine dimesylate on parent-rated measures in children aged 6 to 12 years with attention-deficit/hyperactivity disorder: a secondary analysis. Postgrad Med. 2008;120(3):89-102.

31. Jain R, Babcock T, Burtea T, et al. Efficacy of lisdexamfetamine dimesylate in children with attention-deficit/hyperactivity disorder previously treated with methylphenidate: a post hoc analysis. Child Adolesc Psychiatry Ment Health. 2011;5(1):35.

32. Faraone SV, Buitelaar J. Comparing the efficacy of stimulants for ADHD in children and adolescents using meta-analysis. Eur Child Adolesc Psychiatry. 2010;19(4):353-364.

33. Faraone SV. Understanding the effect size of lisdexamfetamine dimesylate for treating ADHD in children and adults. JAtten Disord. 2012; 16(2):128-137.

34. Wigal SB, Kollins SH, Childress AC, Adeyi B. Efficacy and tolerability of lisdexamfetamine dimesylate in children with attention-deficit/ hyperactivity disorder: sex and age effects and effect size across the day. Child Adolesc Psychiatry Ment Health. 2010;4:32-49.

35. Findling RL, Childress AC, Krishnan S, McGough JJ. Long-term effectiveness and safety of lisdexamfetamine dimesylate in school-aged children with attention-deficit/hyperactivity disorder. CNS Spectr. 2008;13(7):614-620.

36. Findling RL, Ginsberg LD, Jain R, Gao J. Effectiveness, safety, and tolerability of lisdexamfetamine dimesylate in children with attentiondeficit/hyperactivity disorder: an open-label, dose-optimization study. J Child Adolesc Psychopharmacol. 2009;19(6):649-662.

37. Turgay A, Ginsberg L, Sarkis E, et al. Executive function deficits in children with attention-deficit/hyperactivity disorder and improvement with lisdexamfetamine dimesylate in an open-label study. J Child Adolesc Psychopharmacol. 2010;20(6):503-511.

38. Wigal SB, Maltas S, Crinella F, et al. Reading performance as a function of treatment with lisdexamfetamine dimesylate in elementary school children diagnosed with ADHD. J Atten Disord. 2012;16(1):23-33.

39. Findling RL, Childress AC, Cutler AJ, et al. Efficacy and safety of lisdexamfetamine dimesylate in adolescents with attention-deficit/ hyperactivity disorder. J Am Acad Child Adolesc Psychiatry. 2011; 50(4):395-405.

40. Childress AC. Long-term safety and effectiveness of lisdexamfetamine dimesylate in adolescents with attention-deficit/hyperactivity disorder. Proceedings of the 164th Annual Meeting of the American Psychiatric Association; May 14-18, 2011; Honolulu, HI.
41. Childress AC, Cutler AJ, Saylor K, et al. Participant-perceived quality of life in a long-term open-label trial of lisdexamfetamine dimesylate in adolescents with attention-deficit/hyperactivity disorder. Proceedings of the American Academy of Child and Adolescent Psychiatry and Canadian Academy of Child and Adolescent Psychiatry Joint Annual Meeting; October 18-23, 2011; Toronto, Canada.

42. Coghill DR, Tobias B, Lecendreux ML, et al. Efficacy and safety of lisdexamfetamine dimesylate in children and adolescents with attentiondeficit/hyperactivity disorder: a Phase 3 , randomized, double-blind, multicenter, parallel-group, placebo-and-active-controlled, doseoptimization study in Europe. Proceedings of the American Academy of Child and Adolescent Psychiatry and Canadian Academy of Child and Adolescent Psychiatry Joint Annual Meeting; October 18-23, 2011; Toronto, Canada.

43. Katic A, Ginsberg L, Jain R, et al. Clinically relevant changes in emotional expression in children with ADHD treated with lisdexamfetamine dimesylate. J Atten Disord. December 20, 2011. [Epub ahead of print].

44. Faraone SV, SpencerTJ, Kollins SH, Glatt SJ. Effects of lisdexamfetamine dimesylate treatment for ADHD on growth. J Am Acad Child Adolesc Psychiatry. 2010;49(1):24-32.

45. MTA Cooperative Group. A 14-month randomized clinical trial of treatment strategies for attention deficit/hyperactivity disorder. Arch Gen Psychiatry. 1999;56(12):1073-1086.

46. Wigal SB, Jun A, Wong AA, Stehli A, Steinberg-Epstein R, Lerner MA. Does prior exposure to stimulants in children with ADHD impact cardiovascular parameters from lisdexamfetamine dimesylate? Postgrad Med. 2010;122(5):27-34.

47. Cooper WO, Habel LA, Sox CM, et al. ADHD drugs and serious cardiovascular events in children and young adults. $N$ Engl J Med. 2011; 365(20):1896-1904.

48. Vetter VL, Elia J, Erickson C, et al. Cardiovascular monitoring of children and adolescents with heart disease receiving medications for attention deficit/hyperactivity disorder: a scientific statement from the American Heart Association Council on Cardiovascular Disease in the Young Congenital Cardiac Defects Committee and the Council on Cardiovascular Nursing. Circulation. 2008;117(18): $2407-2423$.

49. O'Keefe L. ECGs for all ADHD patients? AAP-AHA release joint "clarification" on AHA recommendation. AAP News. 2008;29(6):1.

50. Giblin JM, Strobel AL. Effect of lisdexamfetamine dimesylate on sleep in children with ADHD. JAtten Disord. 2011;15(6):491-498.

51. Spiller HA, Griffith JR, Anderson DL, Weber JA, Aleguas A. Poison centers detect an unexpectedly frequent number of adverse drug reactions to lisdexamfetamine. Ann Pharmacother. 2008;42(7):1142-1143.

52. Brahm NC, Hamilton DR. Alopecia following initiation of lisdexamfetamine in a pediatric patient. Prim Care Companion J Clin Psychiatry. 2009;11(6):365.

53. Hood B, Nowicki MJ. Eosinophilic hepatitis in an adolescent during lisdexamfetamine dimesylate treatment for ADHD. Pediatrics. 2010; 125(6):e1510-e1513.

54. Goodman DW, Scheckner B, Dirks B, et al. Safety profile of lisdexamfetamine dimesylate in clinical trials in children, adolescents, and adults with attention-deficit/hyperactivity disorder. Proceedings of the 163rd Annual Meeting of the American Psychiatric Association; May 22-26, 2011; New Orleans, LA.

55. Wigal SB, Chae S, Patel A, Steinberg-Epstein R. Advances in the treatment of attention-deficit/hyperactivity disorder: a guide for pediatric neurologists. Semin Pediatr Neurol. 2010;17(4):230-236.

56. Arnold LE. Methylphenidate vs amphetamine: comparative review. J Atten Disord. 2000;3(4):200-211.

57. Jasinski DR, Krishnan S. Abuse liability and safety of oral lisdexamfetamine dimesylate in individuals with a history of stimulant abuse. J Psychopharmacol. 2009;23(4):419-427.

58. Jasinski DR, Krishnan S. Human pharmacology of intravenous lisdexamfetamine dimesylate: abuse liability in adult stimulant abusers. J Psychopharmacol. 2009;23(4):410-418. 
59. Khoza S, Oladapo AO, Barner JC. Adherence to medication for attention/deficit/hyperactivity disorder: does time frame matter? J Pharm Health Serv Res. 2011;2(3):157-163.

60. Patrick DL, Edwards TC, Topolski TD. Adolescent quality of life, part II: initial validation of a new instrument. J Adolesc. 2002;25(3): $287-300$.
61. Antonucci D, Kunins C, Manos M, Lopez FA, Kerney DL. Assessing effects of treatment with lisdexamfetamine dimesylate for pediatric ADHD using a parental survey. CNS Spectr. 2010;15(4):248-256.

\section{Publish your work in this journal}

Adolescent Health, Medicine and Therapeutics is an international, peer-reviewed, open access journal focusing on health, pathology, and treatment issues specific to the adolescent age group. All aspects of health maintenance, preventative measures and disease treatment interventions are addressed within the journal and practitioners from all disciplines are invited to submit their work as well as healthcare researchers and patient support groups.. The manuscript management system is completely online and includes a very quick and fair peerreview system. Visit http://www.dovepress.com/testimonials.php to read real quotes from published authors.

Submit your manuscript here: http://www.dovepress.com/adolescent-health-medicine-and-therapeutics-journal 